\title{
A CORRECTION TO MY PAPER “ON THE NON- COMMUTATIVITY OF PONTRJAGIN RINGS \\ MOD 3 OF SOME COMPACT EXCEPTIONAL GROUPS"'
}

\author{
SHÔRÔ ARAKI
}

This note is a correction of an error of the author's paper mentioned in the title. (The reference [1]). The proof of the Prop. 6 of [1], Chap. II, p. 247 , is an error. And the propositions and formulas in pp. 247-249 of [1] depending on this Prop. 6 must be corrected. All notations are referred to [1].

1. We continue the discussion of $[1$, p. 246]. The singular planes of $Q$ are partially ordered by the ordering of associated planes in $P$. Give a linear order in $\boldsymbol{Q}$ compatibly with this partial order. Then any subsequence $Q_{k}$ of length $k$ gives a $2 k$-dimensional sub $E_{6}$-cycle $\Gamma\left(Q_{k}\right)$ of $\Gamma(f P)$. The totality of these $2 k$-dimensional $E_{6}$-cycles forms an additive basis of $H_{2 k}(\Gamma(f P): Z)$. The dual cohomology class of $\Gamma\left(Q_{k}\right)$ is $y_{i_{1}}^{\left(\varepsilon_{1}\right)} \ldots y_{i_{k}}^{\left(\varepsilon_{k}\right)}$ for $Q_{k}=\left\{q_{i_{1}}^{\left(\varepsilon_{1}\right)}, \ldots, q_{i_{k}}^{\left(\varepsilon_{k}\right)}\right\}$ where $\varepsilon_{s}=0$ if $\rho_{i_{s}}$ is a long root of $F_{4}$ and $\varepsilon_{s}=1$ or 2 if $\rho_{i_{s}}$ is a short root.

Now the Prop. 6, Chap. II of [1], must be corrected as follows:

Proposition 6. The 2 k-cycles $f_{P} \Gamma(P)$ and

$$
\sum x_{i_{1}} \cdots x_{i_{k}}(\Gamma(P)) \cdot \Gamma\left(\left\{q_{i_{1}}^{\left(\varepsilon_{1}\right)}, \ldots, q_{i_{k}}^{\left(\varepsilon_{k}\right)}\right\}\right)
$$

represent the same class in $H_{2 k}(\Gamma(f P): Z)$, where the summation runs over all subsequences $\left\{q_{i_{1}}^{\left(\varepsilon_{1}\right)}, \ldots, q_{i_{k}}^{\left(\varepsilon_{k}\right)}\right\}$ of length $k$ of $Q$.

Since $f_{P}^{*}\left(y_{i_{1}}^{\left(\varepsilon_{1}\right)} \cdots y_{i_{k}}^{\left(\varepsilon_{k}\right)}\right)=x_{i_{1}} \cdots x_{i_{k}}$ by (11) of $[1$, p. 246], a standard argument proves this proposition immediately. The crucial of the erroneous statement of the Prop. 6 lies in what the author had overseen that the $2 k$ cohomology class such as $\left(x_{2}\right)^{2} x_{3} \cdots x_{k}$ is not necessarily zero in general.

The Prop. 7 of $[1$, p. 547$]$ should be corrected as follows :

Proposition 7. $\Omega f_{*}\left(P_{*}\right)=\sum x_{i_{1}} \cdots x_{i_{k}}(\Gamma(P)) \cdot\left\{q_{i_{1}}^{\left(\varepsilon_{1}\right)}, \ldots, q_{i_{k}}^{\left(\varepsilon_{k}\right)}\right\}_{*}$ where $\Omega f_{*}$ denotes the homology map induced by $\Omega f$ and the summation is the same as in

Received June 23, 1961. 
the Prop. 6.

The proof is entirely the same as in the proof of the Prop. 7 of [1, p. 247].

2. The formula (12) of $[1$, p. 247$]$ is correct as is easily seen from the corrected Prop. 7.

The formulas $\left(12^{\prime}\right)$ and $\left(12^{\prime \prime}\right)$ of $[1$, p. 248] are incorrect. If we compute by making use of the Prop. 4.2 of [2, Chap. III], then we see that the cohomology rings $H^{*}\left(\Gamma\left(P_{5}^{1}\left(F_{4}\right)\right): Z\right)$ and $H^{*}\left(\Gamma\left(P_{5}^{2}\left(F_{4}\right)\right): Z\right)$ have the relations

$$
x_{1}^{2}=0, \quad x_{2}\left(x_{1}+x_{2}\right)=0
$$

among others, and the cohomology fundamental classes are $x_{1} x_{2} x_{3} x_{4} x_{5}$ for both rings. Then the corrected Prop. 7 and the relations $(*)$ prove the following corrections of the formulas $\left(12^{\prime}\right)$ and $\left(12^{\prime \prime}\right)$ :

$\left(12^{\prime}\right)$

$$
\begin{aligned}
& \Omega f_{*}\left(P_{5 *}^{1}\left(F_{4}\right)\right)=P_{5 *}^{1}\left(E_{6}\right)+P_{5 *}^{2}\left(E_{6}\right)-P_{5 *}^{3}\left(E_{6}\right)+\left\{\left(\mu_{4}^{\prime}, 1\right), P_{4}^{1}\right\}_{*}+\left\{\left(\mu_{4}, 1\right), P_{4}^{2}\right\}_{*} \\
& \left.13^{\prime}\right) \quad \Omega f_{*}\left(P_{5 *}^{2}\left(F_{1}\right)\right)=\left\{\left(\mu_{3}-\varphi_{3}^{\prime}, 1\right), P_{4}^{1}\right\}_{*}+\left\{\left(\mu_{3}-\varphi_{3}^{\prime}, 1\right), P_{4}^{2}\right\}_{*}-P_{5 *}^{3}\left(E_{\hat{0}}\right) .
\end{aligned}
$$

The same argument as in pp. 248-249 above the Prop. 8 of [1], with the corrected $\left(12^{\prime}\right)$ and $\left(12^{\prime \prime}\right)$, prove the following corrected formulas of (13) and (14):

$$
\begin{gathered}
\Omega f_{*}\left(P_{5 *}^{1}\left(F_{1}\right)\right)=P_{5 *}^{1}\left(E_{6}\right)+P_{5 *}^{2}\left(E_{6}\right)+P_{5 *}^{3}\left(E_{6}\right), \\
\Omega f_{*}\left(P_{5 *}^{2}\left(F_{4}\right)\right)=P_{5 *}^{3}\left(E_{6}\right) .
\end{gathered}
$$

The Prop. 8 of $\left[1\right.$, p. 249] is exact and the Prop. $8^{\prime}$ is false as is easily seen from the formula (12) and the corrected formulas (13) and (14). We can state the Prop. 8 in a more stronger form as follows:

PROPOSITION 8 ". The homology map $\Omega f_{*}$ is injective in deg $\leqq 10$ for any coefficients.

In the discussion in Chap. III of [1] only the Prop. 8 is refered from pp. 247-249 so that no more related corrections are needed.

3. We can prove the above proposition in its most general form.

The diagram of the symmetric space $E_{6} / F_{4}$ is of type $A_{2}$ and all roots have multiplicity $8([3]$, p. 422$)$. The $K$-cycles of [2] describing the additive basis of $H_{*}\left(\Omega\left(E_{6} / F_{4}\right) ; Z_{2}\right)$ are all iterated 8 -sphere bundles over 8 -spheres, 
whence in particular orientable. Then $H_{*}\left(Q\left(E_{6} / F_{1}\right) ; Z\right)$ has no torsion and $H_{i}\left(\Omega\left(E_{6} / F_{4}\right) ; Z\right)=0$ if $i \neq 0(\bmod 8)$ by [2].

The spectral sequence associated with the fibration $\Omega\left(E_{6}\right) \rightarrow \Omega\left(E_{6} / F_{4}\right)$ (fibre $\Omega\left(F_{4}\right)$ ) is collapsed for any coefficients since odd degree homologies vanish for all three involved homology groups. Hence $\Omega\left(F_{4}\right)$ is totally non homologous zero in $\Omega\left(E_{6}\right)$ for any coefficients, i.e., we obtained the

Proposition. The homology map $\Omega f_{*}: H_{*}\left(\Omega F_{4}: G\right) \rightarrow H_{*}\left(\Omega E_{6}: G\right)$ is injective in all degrees and for any coefficient group $G$.

A related question will be discussed elsewhere.

\section{REFERENCES}

[1] S. Araki, On the non-commutativity of Pontrjagin rings mod 3 of some compact exceptional groups, Nagoya Math. J., 17 (1960), 225-260.

[2] R. Bott and H. Samelson, Applictions of the Theory of Morse to Symmetric Spaces, Amer. J. Math., 80 (1958), 964-1029.

[3] E. Cartan, Sur certaines formes Riemanniens remarquables des géométries à groupe fondamental simple, Ann. Sci. de l'Ecole Normale Supérieure, 44 (1927), 345-467.

Kyusyu University. 